\title{
Congruência por Corte e Terceiro Problema de Hilbert
}

\author{
Scissors Congruence and Hilbert's Third Problem \\ Parham Salehyan ${ }^{1}$ e Ronaldo Dias ${ }^{2}$ \\ 1,2 Universidade do estado de são paulo, Unesp - São José do Rio Preto, SP, Brasil
}

\begin{abstract}
Resumo
Dados dois polígonos com áreas iguais, épossível decompor um deles em um número finito de polígonos e reconstruir o outro. Esse fato é conhecido como teorema de Bolyai-Gerwien. É natural perguntarmos se este teorema é válido para poliedros com volumes iguais. Essa pergunta inicialmente proposta por Bolyai e Gauss, em 1844, e depois pelo Hilbert como o Terceiro Problema na sua famosa lista de 23 problemas, foi respondida negativamente por Max Dehn, em 1902, para poliedros em dimensão três. Nosso objetivo principal é apresentar a prova de Dehn. Este artigo possui duas partes principais. A primeira é dedicada ao conceito de área: faremos uma breve revisão envolvendo alguns fatos históricos até seu formalismo na geometria e provaremos o teorema de Bolyai-Gerwien. Na segunda parte veremos como o conceito de área e suas propriedades podem ser interpretados no espaço euclidiano de dimensão três. O resultado principal é o teorema de Dehn-Hadwiger que é fundamental para resolver o problema de Hilbert para poliedros.
\end{abstract}

Palavras-chave: Área e Volume. Poliedros e Polítopos. Terceiro Problema de Hilbert

\begin{abstract}
Given two polygons with equal areas, one can decompose one of them into a finite number of polygons and rebuild another. This fact is known as the theorem of Bolyai-Gerwien. It is natural to ask whether this theorem is true for polyhedra with equal volumes. This question originally proposed by Bolyai and Gauss in 1844 and then by Hilbert as the Third Problem in his famous list of 23 problems, was negatively answered by Max Dehn in 1902 for polyhedra in three dimension. The main objective of this paper is to present the proof of Dehn. This article has two main parts. The first one is devoted to the concept of area: we will briefly review involving some historical facts to his formalism in geometry and prove the theorem of Bolyai-Gerwien. In the second part we will see how the concept of area and its properties can be interpreted in Euclidean space of dimension three. The main result is the theorem of Dehn-Hadwiger which is crucial to solve the problem of Hilbert for polyhedra.
\end{abstract}

Keywords: Area and Volume. Polyhedra and Polytopes. Third Hilbert's Problem 


\section{Introdução: Área e Congruência por Corte}

A noção de medida ou mais precisamente área e volume para figuras geométricas apareceu há mais de 5000 anos pela necessidade de saber suas medidas e compará-las. Nos tempos antigos, como atualmente, os impostos sobre as terras de agricultores eram cobrados de acordo com suas áreas e acreditava-se que se dois pedaços de terra tivessem o mesmo formato, teriam a mesma medida. Esse tipo de problema, entre outros, fez a Geometria nascer. De fato, nos tempos antigos, a geometria era sinônimo de medir a terra.

Conceitos como comprimento, área e volume embora muito antigos e fundamentais na geometria, são difíceis de serem definidos precisamente em matemática Os esforços para definir e generalizar esses conceitos levaram ao aparecimento de uma teoria em matemática hoje em dia chamada de teoria de medidas. Uma parte dessa dificuldade surge pelo fato de que os subconjuntos do plano e espaço são idênticos ou muito parecidos com os objetos do mundo real e também em alguns casos é inevitável usar a teoria de medida. O primeiro passo para definir a área é defini-la para polígonos, uma vez que são as figuras planas mais simples.

Uma abordagem simples para calcular a área de um polígono é escolher um quadrado padrão, como sendo a unidade da área e cobrir o polígono com cópias desse quadrado. Em geral, alguns desses quadrados ficam no interior do polígono e outros possuem partes fora, dando assim cotas superior e inferior para a área do polígono. Para obter a área exata, devemos refinar este processo, isto é, escolher um quadrado padrão cada vez menor, e esperar que essas cotas convirjam para o mesmo número. Esse será a área do polígono. Desta forma construímos a função área: associamos um número real positivo $a(P)$ a um polígono $P$. Por exemplo, observem como obter estas cotas para o triângulo $A B C$ na figura abaixo. Na aproximação no lado esquerdo 3 e
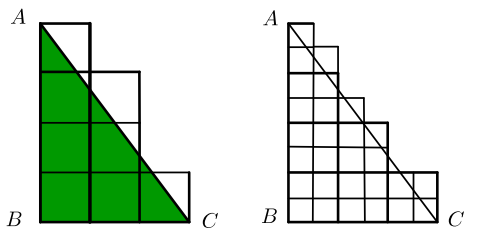

Figura 1: aproximando área.

8 são as cotas inferior e superior para a área e no lado direito as cotas são $18 \times \frac{1}{4}=4,25$ e $28 \times \frac{1}{4}=6,25$.

Esse método, embora não prático e satisfatório para figuras planas mais complicadas, funciona bem para os polígonos. Por outro lado, observamos que, mesmo para polígonos, envolve processos infinitos e argumentos de continuidade. Então seria razoável pensar numa definição que exija técnicas mais elementares. Com esse objetivo, como é comum em matemática, definiremos a função área satisfazendo as propriedades desejáveis e esperadas chamadas de Axiomas de Área:

- Positividade: $a(P)>0$;

- Aditividade: Para dois polígonos disjuntos $P_{1}$ e $P_{2}$, ou seja, se $P_{1} \cap P_{2}$ for vazio ou contiver apenas vértices ou lados de $P_{1}$ e $P_{2}, a\left(P_{1} \cup P_{2}\right)=a\left(P_{1}\right)+$ $a\left(P_{2}\right)$;

- Invariância: Se $P_{1}$ é um polígono e outro polígono $P_{2}$ é obtido a partir de $P_{1}$ com apenas movimentos rígidos, então $a\left(P_{1}\right)=a\left(P_{2}\right)$;

- Para o quadrado padrão $S, a(S)=1$.

É possível demonstrar que existe uma única função definida sobre o conjunto dos polígonos que satisfaz estas propriedades. Uma das consequências desse resultado é obter as fórmulas conhecidas para calcular a área de triângulo e retângulo. Uma prova pode ser encontrada em Shiga e Sunada (1996), capítulo 2. Mais precisamente:

Teorema. Existe uma única função, chamada de função área, que satisfaz as propriedades acima. Em particular a área de um retângulo é dada por comprimento $\times$ largura.

Se um polígono $P$ for decomposto em polígonos disjuntos $P_{1}, \ldots, P_{n}$, escrevemos $P=P_{1}+\cdots+P_{n}$.

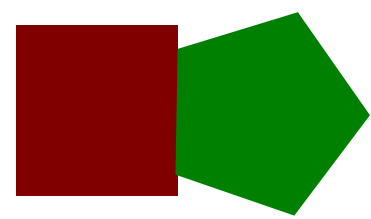

Figura 2: polígonos disjuntos.

Definição. Diremos que dois polígonos $P$ e $Q$ são congruentes por corte e escrevemos $P \sim Q$, se $P=P_{1}+\cdots+$ $P_{n}$ e $Q$ possa ser reconstruído a partir de $P_{1}, \ldots, P_{n}$.

Em outras palavras, a definição acima significa que existem decomposições de $P$ e $Q$ que são soluções para o mesmo quebra-cabeça. É fácil verificar que congruência por corte é uma relação de equivalência. Pela aditividade e invariância da função área, dois polígonos congruentes por corte possuem a mesma área. A recíproca desse fato é conhecido como teorema de BolyaiGerwien. A seguir, demonstraremos dois lemas que serão utilizados para demonstrar esse teorema. 
Lema 1. Todo triângulo é congruente por corte a um retângulo.

Demonstração. Veja a figura 3. Dado o triângulo $A B C$, faremos a seguinte construção. Considere a altura $A H$ e seu ponto médio $P$ e construa o retângulo $B C D E$ tal que $D E$ passe por $P$. Pela construção, obtemos dois pares de triângulos congruentes: $(A P S, B E S)$ e $(A P R, C D R)$ e consequentemente concluímos que o triângulo $A B C$ e o retângulo $B C D E$ são congruentes por corte.

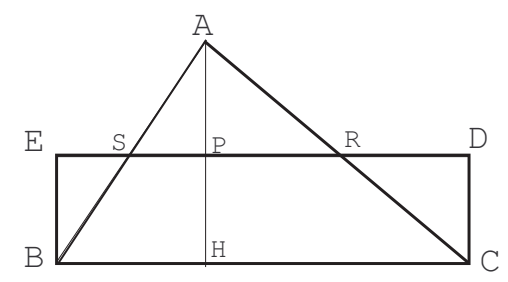

Figura 3: Lema 1.

Uma consequência imediata do lema 1 é a igualdade das áreas do triângulo $A B C$ e retângulo $B C D E$. De fato, pela congruência de triângulos $A P S$ com $B E S$ e $A P R$ com $C D R, a(A P S)=a(B E S)$ e $a(A P R)=a(C D R)$. Pela aditividade e invariância da função área,

$$
\begin{aligned}
a(A B C) & =a(A P S)+a(A P R)+a(S P H B)+a(P R C H) \\
& =a(B E S)+a(C D R)+a(S P H B)+a(P R C H) \\
& =a(B C D E) .
\end{aligned}
$$

Pela construção da demonstração do lema 1 e o teorema da existência da função área, a área de um triângulo é dada por $\frac{\text { base } \times \text { altura }}{2}$. Usando a semelhança entre triângulos, é fácil verificar que este valor é independente da escolha da base e sua altura correspondente.

O próximo lema é de fato o teorema de Bolyai-Gerwien para retângulos.

Lema 2. Dois retângulos com áreas iguais são congruentes por corte.

Demonstração. Considere a figura 4 e a montagem dos retângulos $A B C D$ e $D E F G$. A seguir utilizamos a igualdade das áreas e semelhança dos triângulos $A P E$ e $E C D$ para mostrar $A P=G C$, e consequentemente a congruência dos triângulos APE e QCG. Os triângulos $A P E$ e $E C D$ são retângulos e possuem o ângulo $E$ em comum, portanto são semelhantes, $\log \frac{A P}{D C}=\frac{E A}{E D}$ ou

$$
\frac{A P}{D C}=\frac{E D-A D}{E D} \Rightarrow A P=\frac{D C \cdot E D-D C \cdot A D}{E D} .
$$

Pela igualdade das áreas dos retângulos,

$$
D C \cdot A D=E D \cdot D G,
$$

então

$$
A P=\frac{D C \cdot E D-E D \cdot D G}{E D} \Rightarrow A P=D C-D G=G C .
$$

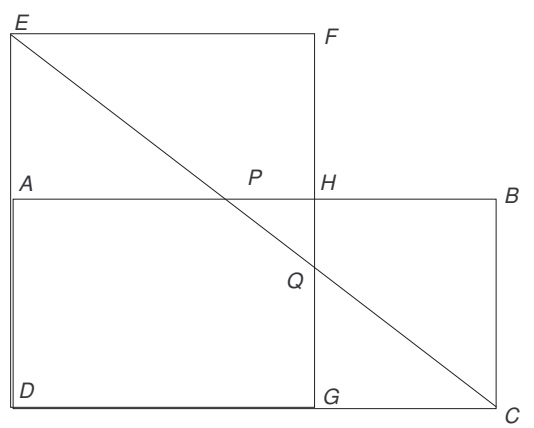

Figura 4: congruência de retângulos.

Analogamente concluímos que os triângulos $E F Q$ e $B C P$ são congruentes. Então

$$
\begin{aligned}
A B C D & =A P Q G D+B C P+Q C G \\
& =A P Q G D+E F Q+A P E \\
& =E F G D .
\end{aligned}
$$

Devemos observar que esta decomposição e o argumento acima funcionam quando os retângulos não são muito compridos. Sem perda de generalidade, suponhamos $B C<E F \leq E D<A B$. Então a construção acima funciona se $A B \leq 2 E D$. Caso contrário, podemos decompor o retângulo com comprimento maior em dois retângulos menores repetidamente até que a condição $A B \leq 2 E D$ seja satisfeita.

A seguir aplicaremos os lemas 1 e 2 para mostrar o teorema de Bolyai-Gerwien que garante a equivalência de congruência por corte e igualdade das áreas dos poligónos.

Teorema de Bolyai-Gerwien. Dois polígonos são congruentes por corte, se, e somente se, possuem a mesma área.

Demonstração. Pela aditividade e invariância da função área, se dois polígonos são congruentes por corte, possuem a mesma área. Para a recíproca, primeiramente lembre-se de que todo polígono $P$ pode ser decomposto em um número finito de triângulos. Pelo lema 1, cada um destes triângulos é congruente por corte a um retângulo e pelo lema 2, cada um destes retângulos é congruente por corte a um retângulo que possui um lado igual a 1 unidade de medida. Sejam $R_{1}, \ldots, R_{n}$ estes retângulos, então

$$
P \sim R_{1}+\cdots+R_{n} .
$$

Construa o retângulo $R$ por meio de encadeamento de $R_{1}, \ldots, R_{n}$. Observe que $R$ possui um lado igual a $1 \mathrm{e}$ outro igual a $a(P)$. Desta forma, concluímos que se $P$ e $Q$ são dois polígonos com a mesma área, ambos são congruentes por corte a $R$, e consequentemente são congruentes por corte (veja Figura 5 a seguir). 


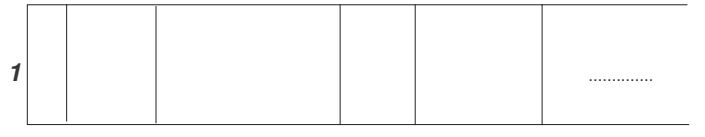

Figura 5: encadeamento de retângulos.

\section{Volume e Congruência por Corte}

Depois de estudar os conceitos de área e congruência por corte para polígonos, é natural pensar em desenvolver uma teoria para volume de poliedros. Inicialmente observamos que os axiomas de área podem ser transformados naturalmente em Axiomas de Volume. Mais precisamente, a função volume $v$ satisfaz:

- Positividade: $v(P)>0$, para todo poliedro $P$;

- Aditividade: Para dois poliedros disjuntos $P_{1}$ e $P_{2}$, ou seja, se $P_{1} \cap P_{2}$ for vazio ou contiver apenas vértices, arestas ou faces de $P_{1}$ e $P_{2}, v\left(P_{1} \cup P_{2}\right)=$ $v\left(P_{1}\right)+v\left(P_{2}\right)$;

- Invariância: Se $P_{1}$ é um poliedro e outro poliedro $P_{2}$ é obtido a partir de $P_{1}$ com apenas movimentos rígidos, então $v\left(P_{1}\right)=v\left(P_{2}\right)$;

- Para o cubo padrão $S, v(S)=1$.

O conceito de congruência por corte também é definido da maneira similar ao caso de polígonos. Se um poliedro $P$ for decomposto em poliedros disjuntos $P_{1}, \ldots, P_{n}$, escrevemos $P=P_{1}+\cdots+P_{n}$. Pela aditividade e invariância da função volume, dois poliedros congruentes por corte possuem o mesmo volume. É natural perguntarmos se vale a recíproca, ou seja, a versão de teorema de Bolyai-Gerwien para os poliedros. Esta pergunta foi proposta pela primeira vez em 1844 por Bolyai e Gauss e depois, em 1900, no Congresso Internacional de Matemáticos, em Paris, por Hilbert. Nesse congresso, Hilbert apresentou uma lista com 23 problemas em aberto naquela época que julgava serem de grande relevância e esta pergunta apareceu como o terceiro problema. Da maneira que ele propôs o problema, era claro que não esperava por uma resposta positiva. Um de seus alunos, Max Dehn, estudou o problema e, em 1902, deu a resposta negativa. De fato, ele mostrou que um cubo e um tetraedro regular de mesmo volume não são congruentes por corte. A seguir, apresentaremos a prova de Dehn. O ponto crucial da demonstração de Dehn é construir invariantes que depois dele foram chamados de Invariantes de Dehn.

Esse invariante é definido por meio de uma função real Q-linear, ou seja, funções $f: \mathbb{R} \rightarrow \mathbb{R}$ tais que $f\left(s_{1}+\right.$ $\left.q s_{2}\right)=f\left(s_{1}\right)+q f\left(s_{2}\right)$ para todo $s_{1}, s_{2} \in \mathbb{R}$ e $q \in \mathbb{Q}$, em outras palavras, transformações lineares de $\mathbb{R}$ visto como Q-espaço vetorial.

Definição. Sejam $P$ um poliedro e $E$ o conjunto de suas arestas. Denote o comprimento da aresta $e$ por $l(e)$ e o ângulo diedro entre duas faces cuja aresta em comum é $e$ por $\theta(e)$. Dada uma função real Q-linear $f$ tal que $f(\pi)=0$, o invariante de Dehn de $P$ associado a $f$ é definido por

$$
D_{f}(P)=\sum_{e \in E} l(e) \cdot f(\theta(e)) .
$$

A ideia dessa definição, a condição $f(\pi)=0$ e o fato que $D_{f}$ define um invariante no conjunto dos poliedros ficarão claros no próximo teorema.

Definição. Seja $S$ um conjunto de números reais. Diremos que $S$ é linearmente independente sobre $\mathbb{Q}$, se

$$
r_{1} s_{1}+\cdots+r_{n} s_{n}=0,
$$

para todo número natural $n, s_{1}, \ldots, s_{n} \in S$ e $r_{1}, \ldots, r_{n} \in$ $\mathrm{Q}$, implica que $r_{1}=\cdots=r_{n}=0$.

Da álgebra linear sabemos dado $S \subseteq \mathbb{R}$ linearmente independente sobre $\mathbb{Q}$, existe uma base $B$ para $\mathbb{R}$ sobre $Q$, tal que $S \subseteq B$. Utilizando este fato, dada uma função real $Q$-linear $f$ com valores definidos em $S$, podemos estendê-la para uma função real Q-linear $\tilde{f}: \mathbb{R} \rightarrow \mathbb{R}$, tal que $\left.\tilde{f}\right|_{S}=f$. Dado $S=\left\{\alpha_{1}, \ldots, \alpha_{n}\right\} \subseteq \mathbb{R}$, seja $V(S)$ o $Q$-espaço vetorial gerado por $S$, i.é.,

$$
V(S):=\left\{q_{1} \alpha_{1}+\cdots+q_{n} \alpha_{n} \mid q_{1}, \ldots, q_{n} \in \mathbb{Q}\right\} .
$$

Dado um poliedro $P$, denotaremos seu conjunto dos ângulos deidrais e o número $\pi$ por $X_{P}$.

O teorema a seguir é o resultado principal demonstrado por Dehn para responder ao terceiro problema de Hilbert. Nesse teorema ele prova que a igualdade dos invariantes de Dehn de dois poliedros é uma condição necessária para eles serem congruentes por corte, em outras palavras, se dois poliedros possuírem invariantes distintos, não serão congruentes por corte. A parte principal da demonstração é mostrar a aditividade do invariante de Dehn, ou seja, se $P$ é um poliedro tal que $P=P_{1}+\cdots+P_{n}$, então $D_{f}(P)=D_{f}\left(P_{1}\right)+\cdots+$ $D_{f}\left(P_{n}\right)$.

Teorema de Dehn-Hadwiger. Sejam $P$ e $Q$ poliedros, $X_{P} \cup X_{Q} \subseteq X$ e $f: V(X) \rightarrow \mathbb{R}$ uma função $\mathbb{Q}$-linear tal que $f(\pi)=0$. Se $P \sim Q$, então $D_{f}(P)=D_{f}(Q)$.

Demonstração. Sejam $P=P_{1}+\cdots+P_{n}, Y:=X_{P_{1}} \cup \cdots \cup$ $X_{P_{n}} \cup X$ e $g: V(Y) \rightarrow \mathbb{R}$ uma função $\mathbb{Q}$-linear, tal que $\left.g\right|_{V(X)}=f$. A seguir mostraremos que o invariante de Dehn é aditivo, i.é,

$$
D_{g}(P)=D_{g}\left(P_{1}\right)+\cdots+D_{g}\left(P_{n}\right) .
$$

Seja $e$ uma das arestas do polígono $P_{i}$. Analisaremos o efeito da parcela $l(e) g(\theta(e))$ no invariante de Dehn associado a $P$. Há três possibilidades: 
- $e$ está contida numa das arestas de $P$. Neste caso, o ângulo diedral $\theta(e)$ do $P_{i}$ será o mesmo que do $P$, portanto esta parcela aparece em $D_{g}(P)$.

- e está contida numa faces de $P$. Neste caso, a soma dos ângulos diedrais associados a $e$ será $\pi$, mas como $g(\pi)=0$, não haverá efeito em $D_{g}(P)$.

- Finalmente se $e$ tiver contida no interior do $P$, a soma dos ângulos diedrais associados a $e$ será $2 \pi$, mas como $g(2 \pi)=2 g(\pi)=0$, não haverá efeito em $D_{g}(P)$.

Seja $Q=Q_{1}+\cdots+Q_{n}$. Como $P \sim Q$, para todo $i=$ $1, \ldots, n$ existe um único $j=1, \ldots, n$ tal que $P_{i}$ e $Q_{j}$ são congruentes, $\log$ o $D_{g}\left(P_{i}\right)=D_{g}\left(Q_{j}\right)$ e

$$
\begin{aligned}
D_{g}(P) & =D_{g}\left(P_{1}\right)+\cdots+D_{g}\left(P_{n}\right) \\
& =D_{g}\left(Q_{1}\right)+\cdots+D_{g}\left(Q_{n}\right) \\
& =D_{g}(Q) .
\end{aligned}
$$

Para finalizar, lembre-se de que $\left.g\right|_{V(X)}=f$, portanto $D_{f}(P)=D_{f}(Q)$.

\section{Conclusão: Terceiro Problema de Hilbert}

O teorema de Dehn-Hadwiger é o principal resultado para resolver o terceiro problema de Hilbert. Basta calcular os invariantes de Dehn para um cubo e tetraedro regular. Seja $C$ um cubo cuja aresta possui comprimento $s$. Neste caso, $X_{C}=\left\{\frac{\pi}{2}, \pi\right\}$. Portanto para qualquer função Q-linear tal que $f(\pi)=0$,

$$
D_{f}(C)=12 s f\left(\frac{\pi}{2}\right)=0 .
$$

No caso de um tetraedro regular $T$, é fácil verificar que o ângulo diedro é $\alpha=\arccos \frac{1}{3}$. Então $X_{T}=\{\alpha, \pi\}$. Por indução, podemos mostrar que

$$
\cos n \alpha=\frac{a_{n}}{3^{n}}, \text { para todo } n \in \mathbb{N},
$$

onde $a_{n}$ é um número inteiro não divisível por 3, em particular $\cos n \alpha \neq \pm 1$. Este fato é suficiente para provar que $\alpha$ e $\pi$ são linearmente independentes sobre $\mathbb{Q}$, caso contrário, existiriam $p, q \in \mathbb{Z}, q>0$ tais que

$$
\alpha=\frac{p}{q} \pi \Rightarrow q \alpha=p \pi \Rightarrow \cos q \alpha= \pm 1,
$$

que é absurdo. Então podemos considerar uma função Q-linear tal que $f(\alpha)=1$ e $f(\pi)=0$. Seja $l$ o comprimento da aresta de $T$, então

$$
D_{f}(T)=6 l f(\alpha) \neq 0 .
$$

Portanto pelo teorema de Dehn-Hadwiger, um cubo e um tetraedro regular com o mesmo volume não podem ser congruentes por corte, uma vez que possuem invariantes de Dehn diferentes.

\section{Mais comentários}

A congruência por corte pode ser apresentada facilmente aos alunos do ensino médio. Para melhor compreensão dos resultados, podemos verificá-los por meio de trabalhos com material concreto. Algumas sugestões podem ser encontradas no trabalho de conclusão do curso do primeiro autor disponível no endereço bit.profmatsbm.org.br/xmlui/.

Vale observar que o teorema de Dehn-Hadwiger mostra que a igualdade dos invariantes de Dehn é uma condição necessária para dois poliedros com volumes iguais serem congruentes por corte. É natural perguntarmos se essa condição é suficiente também. Ou seja, se dois poliedros possuem o mesmo volume e invariantes de Dehn iguais, será que são congruentes por corte? Sydler (1965), deu resposta positiva a essa pergunta: Sejam $P$ e $Q$ poliedros com volumes iguais e $X_{P} \cup X_{Q} \subseteq X$. Então $P$ e $Q$ são congruentes por corte, se, e somente se, possuem invariantes de Dehn iguais para toda função Q-linear $f$ : $V(X) \rightarrow \mathbb{R}$ tal que $f(\pi)=0$. Observem que a hipótese de igualdade de volumes é necessária, basta lembrar que quaisquer dois cubos possuem invariantes iguais a zero, mas se não tiverem volumes iguais claramente não podem ser congruentes por corte. Poucos anos depois, Jessen (1968) apresentou uma demonstração mais simples para o resultado do Sydler e ainda provou que o mesmo vale para poliedros em $\mathbb{R}^{4}$. O problema para dimensões maiores, ou seja, poliedros em $\mathbb{R}^{n}, n \geq 5$, está em aberto. $\mathrm{O}$ leitor interessado poderá consultar as referências Benko (2007), Do (2006), Shiga e Sunada (1996) e Kellerhals (1999) para mais detalhes.

\section{Referências}

Benko, D., 2007. A new approach to hilbert's third problem. The Mathematical Association of America Monthly 114 (0), 665-676.

Do, N., 2006. Scissors congruence and hilbert's third problem. Austral. Math. Soc. Gaz 0 (2), 81-87.

Jessen, B., 1968. The algebra of polyhedra and the dehnsydler theorem. Math. Scand. 0 (22), 241-256.

Kellerhals, R., 1999. Old and new about hilbert's third problem. In: European women in mathematics(Lossum). pp. 179-187.

Shiga, K., Sunada, T., 1996. A Matehmatical Gift, III The interplay between topology, functions, geometry and algebra, 1st Edition. AMS.

Sydler, J. P., 1965. Conditions nécessaires et suffisantes pour l'équivalence des polyèdres de l'espace euclidien à trois dimensions. Comment. Math. Helv. - (40), 43-80. 